\title{
Walking Perception by Walking Observers
}

\author{
Alissa Jacobs and Maggie Shiffrar \\ Rutgers, The State University of New Jersey, Newark
}

\begin{abstract}
People frequently analyze the actions of other people for the purpose of action coordination. To understand whether such self-relative action perception differs from other-relative action perception, the authors had observers either compare their own walking speed with that of a point-light walker or compare the walking speeds of 2 point-light walkers. In Experiment 1, observers walked, bicycled, or stood while performing a gait-speed discrimination task. Walking observers demonstrated the poorest sensitivity to walking speed, suggesting that perception and performance of the same action alters visual-motion processes. Experiments 2-6 demonstrated that the processes used during self-relative and other-relative action perception differ significantly in their dependence on observers' previous motor experience, current motor effort, and potential for action coordination. These results suggest that the visual analysis of human motion during traditional laboratory studies can differ substantially from the visual analysis of human movement under more realistic conditions.
\end{abstract}

Extensive research has been devoted to understanding the visual analysis of human movement. With the use of point-light displays, researchers have repeatedly demonstrated the visual system's keen and robust sensitivity to human movement. In classic demonstrations of this sensitivity, human models with points of light attached to their major joints are filmed while they perform various actions. Although only a few points remain visible in the resultant displays, observers can quickly and accurately identify particular human actions, such as dancing and boxing (e.g., Dittrich, 1993; Johansson, 1973), as well as complex psychological attributes, such as deception and emotion (e.g., Brownlow, Dixon, Egbert, \& Radcliffe, 1997; Dittrich, Troscianko, Lea, \& Morgan, 1996; Runeson \& Frykholm, 1981, 1983). Furthermore, observers can perceptually organize such point-light displays of human action with stimulus durations of only $200 \mathrm{~ms}$ (Johansson, 1976) and with extensive masking (Bertenthal \& Pinto, 1994; Cutting, Moore, \& Morrison, 1988; Thornton, Pinto, \& Shiffrar, 1998).

Thus, prior research has demonstrated that observers have an impressive ability to detect and interpret the motions of other people. It is interesting to note that nearly all of this research has focused on the visual analysis of human movement by stationary observers. As a result, very little is known about how observers analyze the movements of other people for the purpose of action coordination. Although there are many realistic situations in which stationary observers passively analyze the movements of other

Alissa Jacobs and Maggie Shiffrar, Department of Psychology, Rutgers, The State University of New Jersey, Newark.

Some of the results reported in this article were presented at the 44th Annual Meeting of the Psychonomic Society, Vancouver, British Columbia, Canada, November 2003, and the Fourth Annual Meeting of the Vision Sciences Society, Sarasota, Florida, April-May 2004. This research was funded by National Institutes of Health Grant EY12300. We thank Catherine Reed for her valuable assistance with this project.

Correspondence concerning this article should be addressed to Maggie Shiffrar, Department of Psychology, Rutgers, The State University of New Jersey, 301 Smith Hall, Newark, NJ 07102. E-mail: mag@psychology rutgers.edu people - such as when one sits on a park bench and watches children play tag-in many situations, observers actively participate in the actions that they observe. For example, upon joining a game of tag, you must compare your own movements with those of the people around you. We conducted the current set of experiments to better understand the perceptual component of this comparative process.

Previous research has revealed significant interactions between action execution and action perception. In these studies, observers viewed displays of human action or postures while moving their own bodies (e.g., Prinz, 1997; Reed \& Farah, 1995). Such studies have shown that under varying conditions, action performance significantly affects observers' memories and percepts of human bodies and actions. Commensurate with this, other research has demonstrated that action perception can interfere with action production. For example, action execution is disrupted when observers view computer displays of human movement but not when they view displays of robotic movement (Kilner, Paulignan, \& Blakemore, 2003). These findings suggest that a reciprocal linkage exists between action execution and action observation (e.g., Prinz, 1997; Shiffrar \& Pinto, 2002; Viviani \& Stucchi, 1992). The basic premise of such research is that action perception and action production share identical representational or processing codes (see Knoblich \& Flach, 2003, for review). In other words, the same body schema or motor information that is used during action performance also influences action perception. Indeed, neurophysiological and behavioral evidence from studies with human and nonhuman primates has accumulated in support of this assumption (e.g., Rizzolatti, Fadiga, Gallese, \& Fogassi, 1996; Stevens, Fonlupt, Shiffrar, \& Decety, 2000). For instance, mirror neurons selectively respond to the performance and observation of the same action.

Although growing evidence supports the existence of perception-action coupling, it is unclear how moving observers might use such a mechanism when they interact with other people. Research in ecological perception provides some insights. Researchers in this field have identified fundamental linkages between observers' actions and their perceptions of the environments 
in which those actions occur (e.g., Adolph, 2000; E. J. Gibson \& Pick, 2000; J. J. Gibson, 1986; Proffitt, Bhalla, Gossweiler, \& Midgett, 1995). From this perspective, visual analyses are not simply defined by environmental input but are constrained by how observers' actions and experiences relate to that input. Indeed, both present and past motor experience are thought to shape visual perception. For instance, developmental research has demonstrated that infants' percepts of hill safety improve as their crawling experience increases (Adolph, 2000). However, as infants' locomotor behavior shifts from crawling to walking, their perception of hill safety also changes. Toddlers who have just begun to walk must remap this new motor experience to their visual percepts. Similarly, adults' perceptual judgments of hill slope are most accurate for frequently traversed inclines (Proffitt et al., 1995). Moreover, adult observers' ability to locomote safely on sloped surfaces alters their visual percepts of those slopes (Bhalla \& Proffitt, 1999; Proffitt et al., 1995). Age, fatigue, and physical fitness all significantly affect explicit percepts of hill slope. Such results stress the importance of the motor "self" in visual perception.

In the current set of experiments, we examined the effect of observer movement on the visual analysis of human action. Specifically, moving and stationary observers judged the relative gait speeds of two walkers. In Experiment 1, we examined whether stationary and moving observers judge gait speed differently. In Experiment 2, we investigated whether observer motion differentially affects self-relative and other-relative judgments of gait speed. In Experiments 3-5, observers' motor experiences were varied through changes in gait speed, treadmill incline, and physical fitness. Manipulating the motor experiences of our observers allowed us to conduct a more precise examination of the effects of motor input on action perception. Finally, we designed a sixth experiment to determine whether differences in self- and otherrelative action perception depend on the potential for action coordination.

\section{Experiment 1: Gait-Speed Discrimination by Moving and Stationary Observers}

In the real world, observers move. Conversely, in traditional laboratory studies, observers remain stationary. As a result, little is known about how moving observers perceive human movement. To examine the role of action production in action perception, we had stationary and moving observers perform matched gait-speed discrimination tasks. Specifically, observers compared the relative gait speeds of two point-light walker displays. We asked two questions: First, do moving and stationary observers perceive human movement differently? Second, if they do, does the similarity between observed and performed actions influence the visual analysis of human movement?

To address these questions, we had standing, walking, and cycling observers perform a gait-speed discrimination task. In the standing condition, observers stood on a stationary treadmill while they discriminated the relative gait speeds of two point-light walkers. Observers in the walking condition walked on a moving treadmill while performing the same gait-speed discrimination task with the same point-light walkers. In the cycling condition, observers peddled a stationary bicycle while performing the same gait-speed discrimination task. A comparison of performances by walking and standing observers allowed us to examine the first question - that is, do moving and stationary observers perceive human movement differently? If walking influences walking perception, then observers in the walking and standing conditions should differ in their discriminations of gait speed. Specifically, because perception and action are thought to depend on shared representations or resources (e.g., Prinz, 1997), walking should interfere with walking perception.

The cycling condition allowed us to examine whether observer action per se influences the visual perception of action. Prior research suggests that interactions between action production and action perception depend on the similarity of the actions (e.g., Kilner et al., 2003; Reed \& Farah, 1995). For instance, in studies conducted by Reed and Farah (1995), participants reported whether a model's arm or leg position changed while the participants themselves moved either their arms or their legs. Action production selectively influenced body-position memory. That is, when participants moved their arms, their ability to detect changes in the model's arm, but not leg, positions was affected. Conversely, when participants moved their legs, their ability to detect changes in the model's leg, but not arm, positions was affected. If the similarity between observed and performed actions influences the visual analysis of human movement, then walking and cycling observers should differ in their visual sensitivity to walking speed. Specifically, walking, but not cycling, should interfere with walking perception.

\section{Method}

Participants. Forty-eight undergraduate students from Rutgers University, Newark, participated in this experiment. In this and all other experiments reported in this article, all participants had normal or corrected-tonormal visual acuity and were naive as to the hypothesis under investigation. According to questionnaires completed before participation, all participants were free of any medical conditions that might have prevented them from walking on a treadmill or riding a stationary bicycle.

Apparatus. Stimuli were presented on a G-3 Macintosh computer with a $41-\mathrm{cm}$ monitor. The monitor's resolution was set at $832 \times 624$ pixels, with a refresh rate of $75 \mathrm{~Hz}$. Observers' responses were collected with a Macintosh keyboard. During the experiment, observers either walked or stood on a Quinton Q55 Model 645 treadmill or peddled a Weslo Pursuit 510CS recumbent bicycle.

Stimulus construction. Point-light stimuli were created by filming, with a Cannon Optura digital camera, human actors dressed in black clothing with luminous white hemispheres attached to their major joints. The actors walked on a Quinton Hyperdrive Club Track treadmill at 10 different speeds ranging from 2.0 to $6.5 \mathrm{~km} / \mathrm{hr}$ in $0.5-\mathrm{km} / \mathrm{hr}$ intervals. The distance between the walker and the video camera was approximately $4 \mathrm{ft}$ $(\sim 1.2 \mathrm{~m})$. The actors adopted the most comfortable and energy-efficient gait for each speed. Thus, at each of the 10 speeds, each actor adopted a different combination of step frequency (number of steps per min) and step amplitude (size of step). Because all of our experiments required observers to compare the speeds of two different people, step frequency and step amplitude had to be jointly considered for accurate gait-speed analysis.

The resultant digital movies were exported to a G-3 Macintosh computer. Extraneous background information was filtered out using Adobe Premiere so that only the white markers, with an average diameter of 0.3 $\mathrm{cm}$, remained visible in the final displays. The image size of the resultant Quick Time movies was $320 \times 240$ pixels. From the observer's viewpoint, the average height of the point-light walkers was approximately $3.9^{\circ}$ of visual angle, and the maximum width of each walker, at the moment when 
their hands and feet were maximally separated, was approximately $2.5^{\circ}$ of visual angle.

Procedure. In this experiment, observers compared the speeds of two point-light walkers simultaneously displayed on a computer monitor. The point-light walker presented on the right side of the computer screen was labeled PAT. The point-light walker on the left side of the computer screen remained unlabeled. On each trial, the observer's task was to report whether PAT walked faster or slower than the unlabeled point-light walker. Observers completed eight blocks of 20 trials each. Across blocks, PAT's gait speed varied from 2.5 to $6.0 \mathrm{~km} / \mathrm{hr}$ in $0.5-\mathrm{km} / \mathrm{hr}$ intervals. Within each block of 20 trials, PAT's speed remained constant. On each trial, PAT's gait speed differed by $0.5 \mathrm{~km} / \mathrm{hr}$ from the gait speed of the unlabeled point-light walker. On half of the trials, PAT had the faster gait speed, and on the other half of the trials, PAT had the slower gait speed. Observers reported whether PAT had the faster or slower gait speed by pressing one of two keys on a keyboard fastened to the treadmill control panel.

There were three conditions. In each, the center of the computer monitor was positioned $58 \mathrm{~cm}$ from the observer at eye height. The observer and the point-light walkers always faced in the same direction. Observers turned their heads slightly to the left to view the displays. As depicted in Figure $1 \mathrm{~A}$, observers in the standing condition simply stood on the stationary treadmill as they compared the speeds of two point-light walkers. Observers in the walking condition (see Figure 1B) walked on the treadmill at a constant speed of $4.2 \mathrm{~km} / \mathrm{hr}$ while they compared the gait speeds of the two point-light walkers. This treadmill speed corresponded to the median of all of the gait speeds used in this experiment. In the cycling condition (see Figure 1C), observers peddled the stationary bike at $19 \mathrm{~km} / \mathrm{hr}$ while performing the gait-speed discrimination task. This cycling speed was chosen because it produced heart rates similar to those produced in the walking condition. As such, it controlled for observer exertion level. In all three conditions, observers saw exactly the same displays and performed the same gait-speed discrimination task. The only difference was the action that observers performed.

In this experiment's between-subjects design, 16 observers completed each of three conditions. All observers completed six practice trials before beginning the experimental trials. Only observers who reported feeling comfortable walking on the treadmill or riding the stationary bike while performing the task were allowed to begin the experimental trials. Observers rested as often as needed throughout the experiment.

\section{Results}

We analyzed percentage of correct gait-speed discrimination with a repeated measures analysis of variance (ANOVA). The gait speed of PAT $(2.5-6.0 \mathrm{~km} / \mathrm{hr})$ served as a within-subject variable,
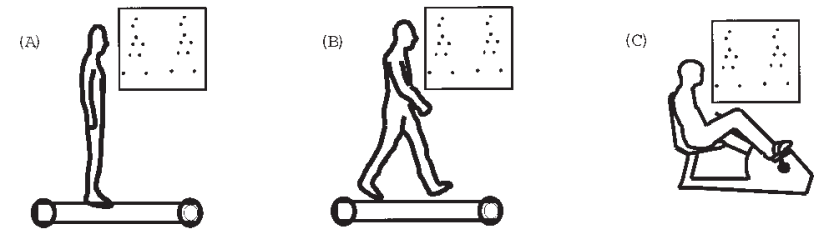

Figure 1. The three conditions of Experiment 1. A: A schematic of a trial used in the standing condition, in which observers viewed two point-light walker displays and judged their relative gait speeds. It is important to note that in this condition, the observer remains stationary while performing the discrimination task. B: A schematic of the walking condition, in which observers walked at a constant pace of $4.2 \mathrm{~km} / \mathrm{hr}$ while judging gait speed. C: A schematic of the cycling condition, in which observers peddled a stationary bike at $19 \mathrm{~km} / \mathrm{hr}$ while performing the same gait-speed discrimination task. and condition (standing, walking, cycling) served as a betweensubjects variable. This analysis revealed a statistically significant main effect of condition, $F(2,45)=4.99, p<.01$. Post hoc analyses revealed that this effect was due to a decrement in performance by walking observers. Specifically, walking observers $(M=61.2 \%)$ demonstrated significantly poorer gait-speed sensitivity than did standing $(M=69.2 \%), t(30)=2.05, p<.05$, or cycling $(M=71.7 \%), t(30)=2.87, p<.05$, observers. Performance in the cycling and standing conditions did not significantly differ, $t(30)=0.91, p=.37$. Overall performance accuracy was significantly above chance in the standing, $t(15)=5.73, p<$ .01 ; walking, $t(15)=3.46, p<.01$; and cycling, $t(15)=12.79$, $p<.01$, conditions. The ANOVA also revealed a statistically significant effect of gait speed, $F(7,315)=10.26, p<.01$. As shown in Figure 2, accuracy decreased as gait speed increased. Consistent with Weber's law, the fixed $0.5-\mathrm{km} / \mathrm{hr}$ difference between the two gait speeds presented on each trial reflected a larger percentage difference for the slower speeds $(0.5 \mathrm{~km} / \mathrm{hr}$ represents $20 \%$ of $2.5 \mathrm{~km} / \mathrm{hr})$ than for the faster speeds $(0.5 \mathrm{~km} / \mathrm{hr}$ represents only $7 \%$ of $6.5 \mathrm{~km} / \mathrm{hr}$ ). A nonsignificant Gait Speed $\times$ Condition interaction revealed that this trend occurred in all three conditions, $F(14,315)=0.31, p>.90$. Thus, decreases in performance with increases in gait speed conform to Weber's law.

\section{Discussion}

Although observers in all three conditions performed exactly the same task, discrimination performances differed significantly. At every gait speed, walking observers performed most poorly. Because only the walking condition interfered with walking perception, it appears that action production influences the perception of similar actions. This is consistent with prior research demonstrating selective interactions between action production and memory of bodily postures (Reed \& Farah, 1995). In this experiment, selective interference of walking production in walking perception may reflect competing demands for access to shared representations (e.g., Prinz, 1997; Rizzolatti et al., 1996) that code for both the execution and perception of the same action.

One could argue that the performance decrement found for walking observers reflects dual-task constraints. In the walking condition, observers simultaneously performed two tasksnamely, gait-speed discrimination and treadmill walking. Dualtask performance has been shown to decrease visual sensitivity to human movement (Thornton, Rensink, \& Shiffrar, 2002). Moreover, walking has been found to inhibit performance on a variety of cognitive tasks (Haggard, Cockburn, Cock, Fordham, \& Wade, 2000). However, because cycling observers performed as well as standing observers did, the performance decrement for walking observers cannot be attributed to dual-task performance. Instead, it appears that walking and standing observers perceive human gait differently.

\section{Experiment 2: Egocentric Versus Exocentric Gait-Speed Discrimination}

The above results suggest that observer movement can significantly influence the visual analysis of point-light displays of human action. Given this, we can now turn to the question of action coordination. Unlike in traditional laboratory studies of 


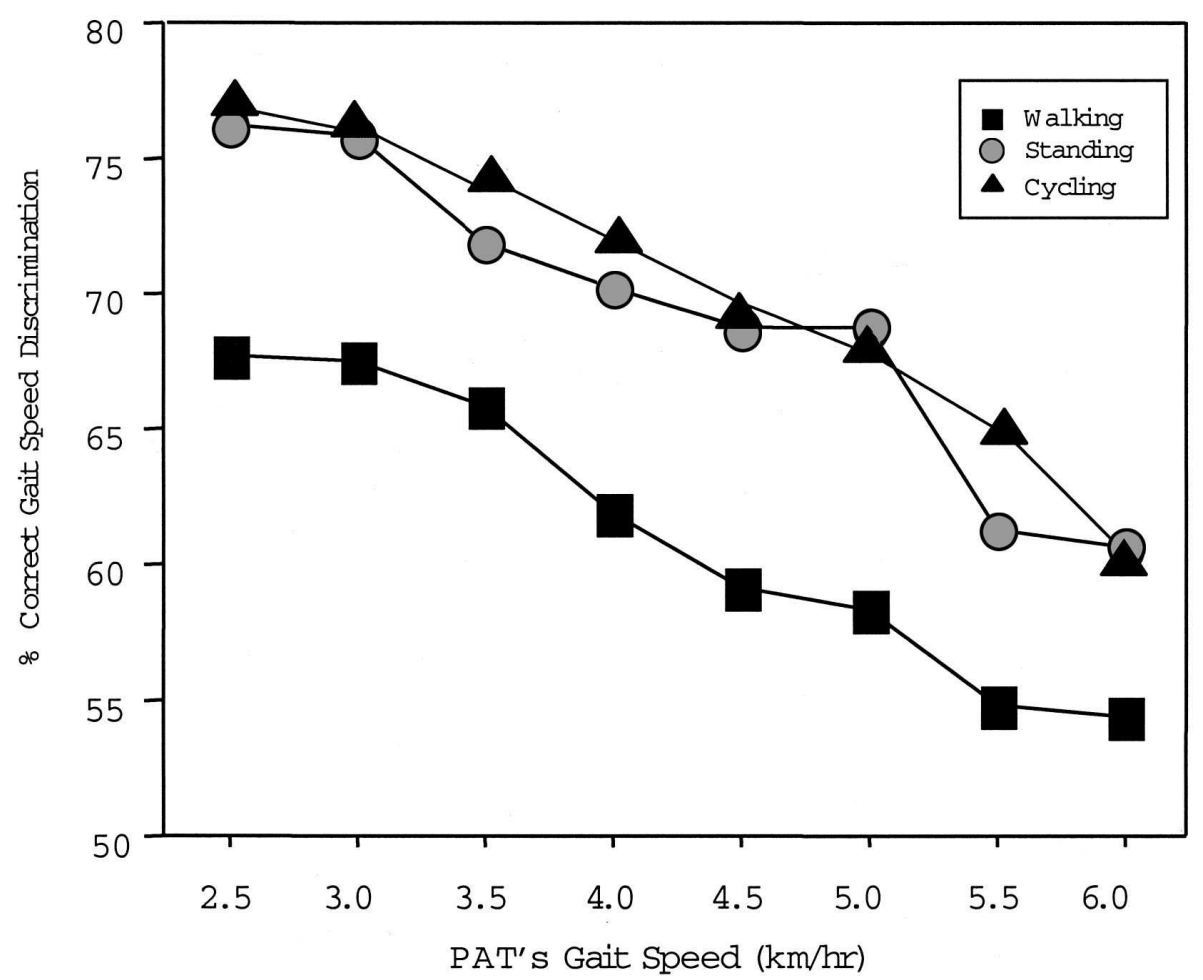

Figure 2. Mean discrimination accuracy in Experiment 1 as a function of condition and gait speed. PAT $=$ the point-light walker presented on the right side of the computer screen.

biological motion perception, under realistic conditions observers frequently analyze human movement so that they can compare their own actions with the actions of other people. For instance, when walking down a street with a friend, you naturally compare your gait with your friend's gait so that you can maintain similar walking speeds and directions. This raises the question of whether walking observers perform self-relative, or egocentric, comparisons of gait speed in the same way that they exocentrically compare the gait speeds of two other people.

Observers clearly use a variety of cues, including optic flow and size (e.g., Cutting, Vishton, \& Braren, 1995; Fajen \& Warren, 2003), to control their gaits within realistic environments. Nonetheless, perceptual comparison of one's own movements with the movements of other people remains a fundamental aspect of action coordination. To learn more about this perceptual process, we had walking observers perform egocentric (self-relative) or exocentric (other-relative) comparisons of gait speeds. Previous research suggests that egocentric and exocentric perceptions can differ. For instance, egocentric judgments of distance, in which observers estimate the distance between themselves and an object, differ significantly from exocentric distance judgments, in which observers estimate the distance between two objects (Loomis, Da Silva, Philbeck, \& Fukusima, 1996). Specifically, egocentric distance perception is more accurate than exocentric distance perception. Egocentric and exocentric percepts of human action are associated with activation of different neural areas (Ruby \& Decety, 2001; Vogeley \& Fink, 2003). Furthermore, behavioral research has identified systematic differences between the perception of one's own actions and perception of the actions of other people (e.g.,
Knoblich \& Flach, 2001, 2003; Loula, Prasad, Harber, \& Shiffrar, 2005). It follows that the ability to compare one's own gait speed with the gait speed of another person may differ from, and even be superior to, one's ability to compare the gait speeds of two other people.

To test this prediction, we extended some previous research concerning prior experience and behavioral relevance in biological motion perception (Jacobs, Pinto, \& Shiffrar, 2004). The results of these earlier experiments confirmed the Gibsonian premise that perceptual sensitivity is defined by behaviorally relevant, but not irrelevant, experience. For example, when observers were asked to perform a behaviorally irrelevant discrimination of the speeds of two distant walkers, performance was independent of the amount of previous experience that observers had had with those gaits. Conversely, when observers performed a behaviorally relevant comparison of the same stimuli-namely, an identity discriminationperformance was experience dependent and reflected greater sensitivity. Thus, behaviorally relevant experience significantly enhances perceptual sensitivity to human gait.

These prior results suggest that egocentric and exocentric judgments of gait speed may differ in their dependence on prior experience. Egocentric action perception may be considered behaviorally relevant because it immediately assists observers in action coordination. For instance, to successfully walk down a street while chatting with a friend, observers must perform finely tuned comparisons of their own gait speed with their friend's gait speed. Conversely, when an observer passively watches people walk by, the relationship between the gait speeds of any two pedestrians is relatively inconsequential to the observer's own 
behavior. Relatedly, research on hill slope and distance perception suggests that perceptual sensitivity is egocentrically defined by one's prior experiences (Adolph, 2000; Bhalla \& Proffitt, 1999; Proffitt et al., 1995; Proffitt, Stefanucci, Banton, \& Epstein, 2003). Together, these findings suggest that egocentric, but not exocentric, gait-speed perception may be experience dependent.

To compare egocentric and exocentric gait-speed perception, we systematically manipulated observer gait speed. Motor research indicates that humans spontaneously, and thus most frequently, adopt gait speeds that are most energetically efficient for their bodies (Hreljac, 1993). On average, these speeds fall between 3.5 and $4.5 \mathrm{~km} / \mathrm{hr}$ for gaits on flat surfaces (Inman, Ralston, \& Todd, 1981). Humans adopt slower (i.e., $2.5-3.0-\mathrm{km} / \mathrm{hr}$ ) or faster (i.e., $5.0-6.0 \mathrm{~km} / \mathrm{hr}$ ) gait speeds far less frequently. Thus, by asking observers in this experiment to walk at normal, faster, and slower gait speeds, we were able to assess whether motor experience influences gait-speed sensitivity under behaviorally relevant conditions. If it does, observers should demonstrate the greatest perceptual speed sensitivity at the most commonly adopted gait speeds.

Further research suggests that egocentric perception is determined by observers' current motor capacities as well as their past motor experiences. For example, decreases in physical fitness and increases in fatigue and age are systematically associated with overestimations of hill steepness (Bhalla \& Proffitt, 1999; Proffitt et al., 1995). By asking observers in the current study to walk at more and less effortful gait speeds, we were able to investigate the role of current motor effort in gait-speed perception. Gait speed and motor exertion are tightly coupled (Hreljac, 1993) because motor effort increases with gait speed. Will it follow that increases in a walking observer's motoric effort will similarly bias his or her visual analysis of gait speed?

\section{Method}

Participants. Thirty-two undergraduate students from Rutgers University, Newark, participated in this experiment for credit toward a psychology class.

Design and procedure. There were two conditions. As depicted in Figure 3A, the exocentric (other-relative) condition was a replication of the walking condition of Experiment 1, except here observers walked at eight different speeds ranging from 2.5 to $6.0 \mathrm{~km} / \mathrm{hr}$. On each trial, the treadmill was adjusted so that observers' gait speeds were identical to the gait speed of PAT, the reference point-light walker on the right side of the display.
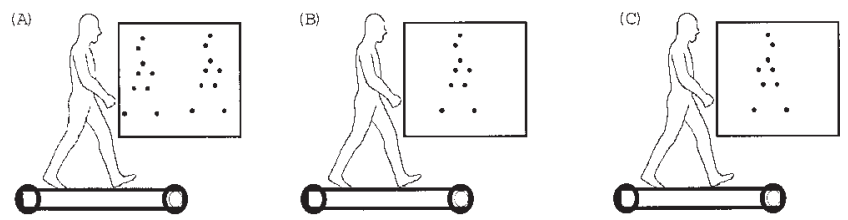

Figure 3. A: A schematic of the exocentric condition of Experiment 2. Walking observers viewed two point-light walker displays and judged the relative gait speeds of the two walkers. B: A schematic of the egocentric condition of Experiment 2. Walking observers viewed one point-light walker and judged their own gait speed relative to the point-light walker's speed. C: A schematic of a trial used in Experiment 6. Observers performed the same egocentric task as in Experiment 2 except with point-light walkers facing in the opposite direction.
However, observers were not informed that their gait speed matched the gait speed of this point-light walker. The unlabeled point-light walker always had a gait speed that was $0.5 \mathrm{~km} / \mathrm{hr}$ faster or slower than PAT's, and thus the observer's, gait speed. As in Experiment 1, on each trial, observers reported whether PAT walked with a faster or slower gait speed than the other point-light walker.

Observers in the egocentric (self-relative) condition adopted the same eight gait speeds. However, rather than view two point-light walker displays on each trial, observers in this condition viewed only one point-light walker (see Figure 3B). The presented walker was identical to the unlabeled walker in the exocentric condition. As in the exocentric condition, the gait speed of this unlabeled point-light walker was $0.5 \mathrm{~km} / \mathrm{hr}$ faster or slower than the observer's gait speed. On each trial, observers in the egocentric condition reported whether they themselves walked faster or slower than the point-light walker. As before, observers recorded their responses by pressing one of two keys on a keyboard fastened to the treadmill control panel.

Thus, in both conditions, observers adopted the same eight gait speeds. Because observers adopted identical gait speeds and performed identical $0.5-\mathrm{km} / \mathrm{hr}$ speed discriminations, the only difference between the egocentric and exocentric conditions was whether observers compared their own speed with the speed of a point-light walker or compared the speeds of two point-light walkers. Observer gait speed ranged from 2.5 to $6.0 \mathrm{~km} / \mathrm{hr}$ in both conditions. Preliminary testing indicated that observers walking at speeds ranging from 3.5 to $4.5 \mathrm{~km} / \mathrm{hr}$ had an average heart rate of 97 beats per minute (bpm). When observers walked more slowly (2.5 and 3.0 $\mathrm{km} / \mathrm{hr}$ ), their heart rate dropped to an average of $89 \mathrm{bpm}$, indicating decreased exertion. Conversely, when observers walked faster than normal, heart rate, and thus motor effort, increased to an average of $117 \mathrm{bpm}$. Thus, by testing observers across different gait speeds, we were able to assess the role of an observer's own motor effort in gait-speed perception.

Half of the observers completed the egocentric condition, and half completed the exocentric condition. At each treadmill speed, observers completed one block of 20 experimental trials. Each point-light walker display was presented 10 times, in random order, during the experiment. On each trial, a fixation point briefly appeared in the center of a computer monitor and was followed by a point-light walker display that remained visible for $3 \mathrm{~s}$.

\section{Results}

We conducted an ANOVA on the data. Observer gait speed $(2.5-6.0 \mathrm{~km} / \mathrm{hr})$ served as a within-subject variable, and condition (egocentric, exocentric) served as a between-subjects variable. A comparison of performance accuracy revealed a significant main effect of condition, $F(1,30)=5.02, p<.05$, and a nonsignificant Observer Gait Speed $\times$ Condition interaction, $F(7,210)=1.87$, $p<.07$. Discrimination accuracy in the egocentric and exocentric conditions produced markedly different patterns of results. As depicted in Figure 4A, consistent with the hypothesis that egocentric judgments should vary as a function of motor experience, performance accuracy in the egocentric condition followed an inverted $U$-shaped function. Performance peaked within the range of the most commonly adopted walking speeds (viz., 3.5-4.5 km/hr; e.g., Hreljac, 1993; Inman et al., 1981). Outside this range, performance decreased. Indeed, accuracy within the range of common walking speeds significantly differed from accuracy for both faster walking speeds $(5.0-6.0 \mathrm{~km} / \mathrm{hr}), t(15)=2.56, p<.05$, and slower walking speeds $(2.5-3.0 \mathrm{~km} / \mathrm{hr}), t(15)=2.78, p<.05$. Performance accuracy in the exocentric condition followed a different pattern, as illustrated in Figure 4A. Here, accuracy conformed to Weber's Law, decreasing with increasing gait speed. 
(A)

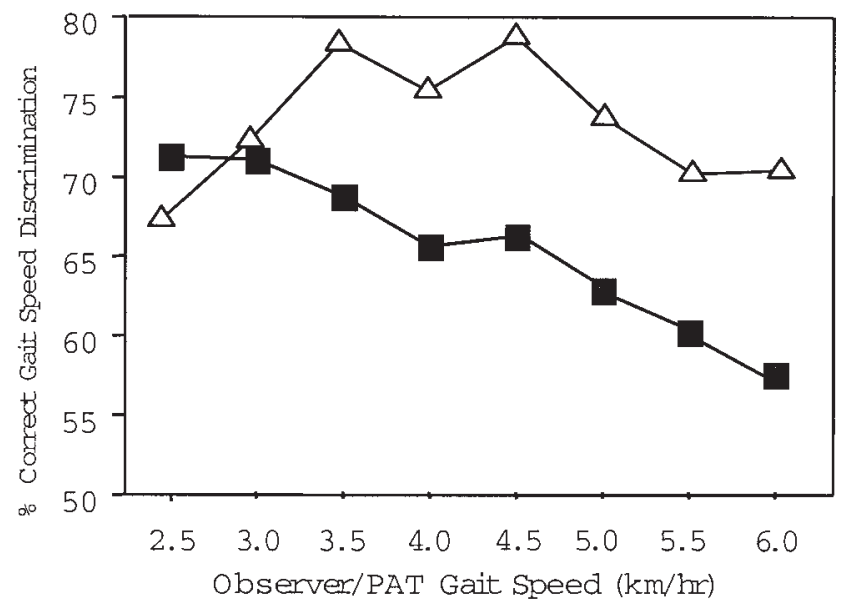

(B)

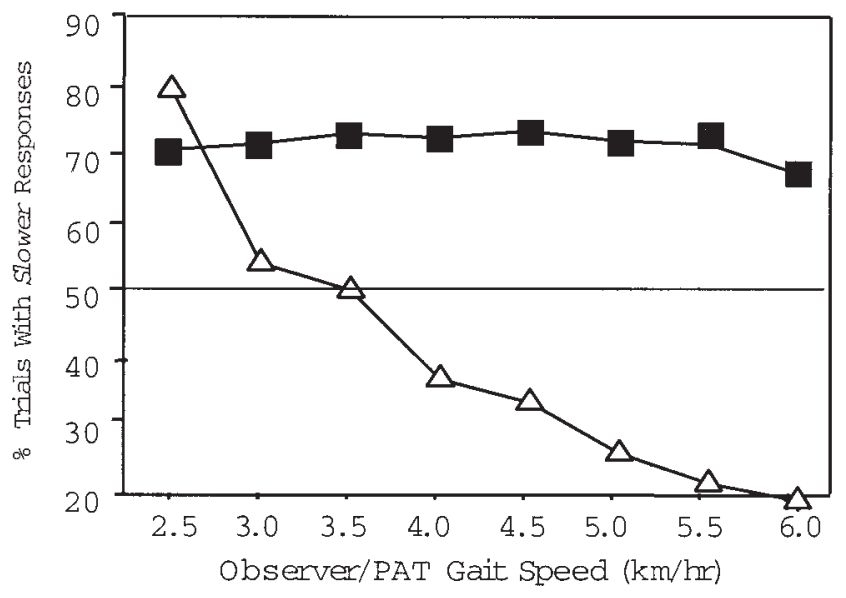

Figure 4. A: Mean discrimination accuracy as a function of condition and gait speed for walking observers performing egocentric and exocentric gait-speed discriminations in Experiment 2. B: Observer bias in Experiment 2 as a function of gait speed and condition. Unbiased responses should fall along the $50 \%$ line.

To better understand performance across conditions, we subsequently analyzed the data and plotted them by response bias. Figure 4B depicts the same data as the percentage of trials during which observers reported that they or PAT walked more slowly than the point-light walker. Unbiased responses should fall along the $50 \%$ line. When bias serves as the dependent measure, dramatic differences are evident between the egocentric and exocentric conditions. Walking observers in the exocentric condition $(M=62.0 \%)$ were significantly more likely to perceive PAT as walking more slowly than the other point-light walker than observers in the egocentric condition were to perceive themselves as walking more slowly than the point-light walker $(M=40.9 \%)$, $F(1,30)=77.59, p<.01$.

Differences in response bias between the egocentric and exocentric conditions can be explained by the significant Observer
Gait Speed $\times$ Condition interaction, $F(7,210)=14.22, p<.01$, illustrated in Figure 4B. Despite the fact that observers in both conditions compared gaits of the same speeds, their performance biases differed dramatically. In the exocentric condition, observers produced a response bias that remained stable across variations in their gait speed, $F(7,15)=0.54, p=.81$. Although observers were not perfectly accurate in their assessments of gait speed, their systematic response biases were unrelated to gait speed. Conversely, perceptual bias in the egocentric condition was directly related to an observer's gait speed, $F(7,15)=30.85, p<.01$. Gait-speed discrimination was significantly less biased at common walking speeds of $3.5-4.5 \mathrm{~km} / \mathrm{hr}(M=42.3 \%)$ than at faster $(M=$ $22.9 \%), t(15)=5.18, p<.01$, and slower $(M=67.3 \%), t(15)=$ $-6.73, p<.01$, walking speeds. At faster walking speeds, observers tended to report walking faster than the point-light walker. Conversely, at slower walking speeds, observers became increasingly likely to report walking more slowly than the point-light walker. It is important to note that this bias pattern was evident not only when observers truly walked more slowly than the point-light walker, $F(7,15)=17.99, p<.01$, but also when they actually walked faster than the point-light walker, $F(7,15)=24.57, p<$ .01 . Because observers in both conditions walked on the same treadmill at the same speeds, observer movement cannot account for differences between egocentric and exocentric perception of human movement.

\section{Discussion}

Although observers in the egocentric and exocentric conditions adopted identical gait speeds and performed equivalent gait-speed comparisons, they produced significantly different patterns of accuracy and response bias. Namely, egocentric gait-speed perception depended on an observer's walking speed, whereas exocentric gait-speed perception did not. This finding is consistent with research in other domains of vision science demonstrating differences between egocentric and exocentric perception (e.g., Loomis et al., 1996). Consistent with the hypothesis that egocentric gait perception should depend on prior walking experience (Jacobs et al., 2004), discrimination performance in the egocentric condition was most accurate at those gait speeds that people most commonly adopt on flat surfaces (Hreljac, 1993; Inman et al., 1981). Thus, egocentric gait-speed perception appears to be experience dependent in the same way that hill slope perception is (Adolph, 2000; Bhalla \& Proffitt, 1999; Proffitt et al., 2003). Conversely, exocentric gait-speed perception appears to be experience independent, at least under the experimental conditions used here.

It is interesting to note that even though the speed difference between the observer and the point-light walker in the egocentric condition remained fixed at $0.5 \mathrm{~km} / \mathrm{hr}$, observers exhibited particular biases that were directly related to the speed at which they walked. Consistent with findings by Proffitt and colleagues that increased effort led to overestimations of hill slope and distance (Bhalla \& Proffitt, 1999; Proffitt et al., 1995, 2003), increases in walking speed, and thus effort, also resulted in overestimations of gait speed. In the same regard, as walking effort decreased with slower treadmill speeds, observers underestimated egocentric walking speed. It is important to note that even though observers in both conditions adopted identical gait speeds, performance was independent of a participant's own gait speed during exocentric 
comparisons, but it was dependent on gait speed during egocentric comparisons. Taken together, these findings suggest that egocentric and exocentric gait-speed perception may rely on different visual-motor processes.

\section{Experiment 3: Egocentric Gait-Speed Sensitivity and Effort}

The findings in Experiment 2 suggest that observer effort can significantly influence observers' perception of the relationship between their own gait speed and the speed of a nearby walker. To examine this effect directly, in this experiment, we modified walking effort by increasing the incline of the treadmill on which observers walked. Research on hill slope perception has found perceptual judgments to vary with the incline of observed hills (Proffitt et al., 1995). At shallow slopes, on which people need not exert much effort to walk up a hill, estimations of hill slope are fairly accurate. Conversely, at steep slopes, on which people must exert substantial effort to walk up a hill, observers' estimations of hill slope are far less accurate, because observers are biased to perceive hills as steeper than they truly are. Because increases in incline are associated with increases in energy expenditure (Diedrich \& Warren, 1998; Susta, Minetti, Moia, \& Ferretti, 2000), raising the treadmill to a $7.5^{\circ}$ incline allowed us to test whether walking effort per se systematically biases egocentric discriminations of gait speed.

Heart rate measures indicated that walking effort was substantially higher on the inclined treadmill $(M=135 \mathrm{bpm})$ used in this experiment than on the flat treadmill $(M=101 \mathrm{bpm})$ used in the previous experiment. If egocentric gait-speed perception is effort dependent, then observers in this experiment should overestimate their own gait speed relative to the speed of the point-light walker's gait.

\section{Method}

Sixteen undergraduate students from Rutgers University, Newark, participated in this experiment for credit toward a psychology class. Observers performed the same gait-speed discrimination task as in the egocentric condition of Experiment 2. Specifically, observers judged whether they walked faster or slower than the point-light walker. The same pairings of observer and point-light gait speeds from Experiment 2 were used. The only difference between this experiment and the egocentric condition of Experiment 2 was the incline of the observers' treadmill. In Experiment 2, the treadmill was flat. In the current experiment, the observers' treadmill was set to a $7.5^{\circ}$ incline. Because it takes more effort to walk at the same speed up a hill than it does on a flat surface (Susta et al., 2000), this incline increased the effort exerted by walking observers.

\section{Results and Discussion}

A one-sample $t$ test revealed that performance accuracy $(M=$ $67.7 \%$ ) in this experiment was significantly above chance, $t(15)=$ $10.51, p<.01$. To better understand the effect of motor effort on gait perception, we conducted an ANOVA comparing performance in this task, in which observers walked up an incline, with performance in the egocentric condition of Experiment 2, in which observers walked on a flat surface. Treadmill incline (flat, inclined) served as a between-subjects variable. Observer gait speed $(2.5-6.0 \mathrm{~km} / \mathrm{hr})$ served as a within-subject variable. Although the analysis of accuracy revealed a nonsignificant main effect of treadmill incline, $F(1,30)=3.39, p<.07$, the analysis did reveal a significant Observer Gait Speed $\times$ Treadmill Incline interaction, $F(7,210)=2.91, p<.01$, as illustrated in Figure 5A. Overall, observers walking up an incline were less accurate in their discriminations of gait speed than were observers walking on a flat surface.

A significant Observer Gait Speed $\times$ Treadmill Incline interaction, $F(7,210)=2.91, p<.01$, indicates that this accuracy decrement was not evident at every speed, as illustrated in Figure 5 A. For common walking speeds $(3.5-4.5 \mathrm{~km} / \mathrm{hr}), t(30)=2.50$, $p<.05$, and faster walking speeds $(5.0-6.5 \mathrm{~km} / \mathrm{hr}), t(30)=2.40$ $p<.05$, discrimination accuracy was significantly lower for observers on the inclined treadmill than for observers on the flat

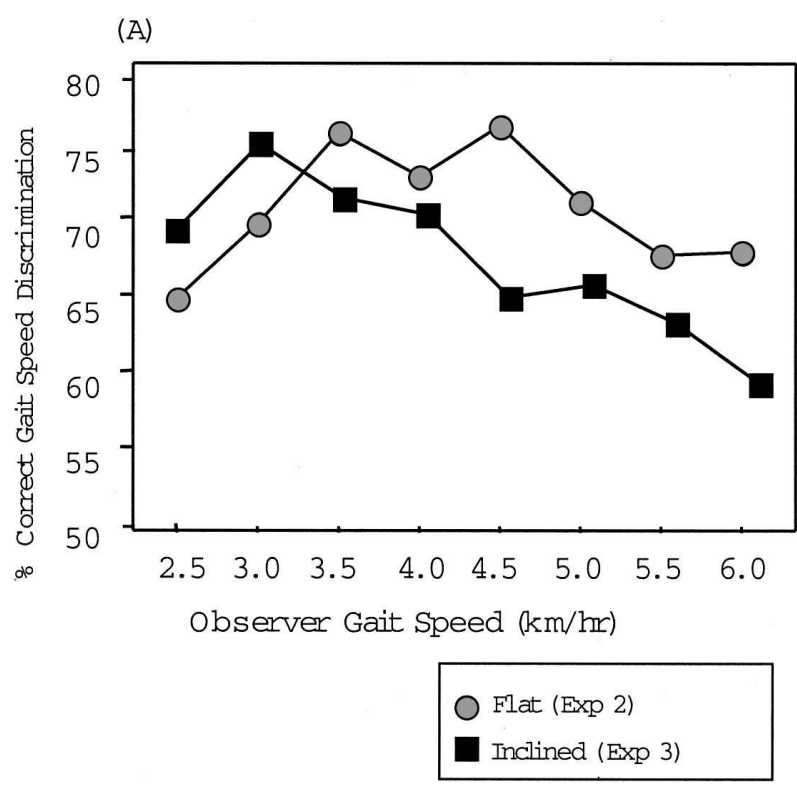

(B)

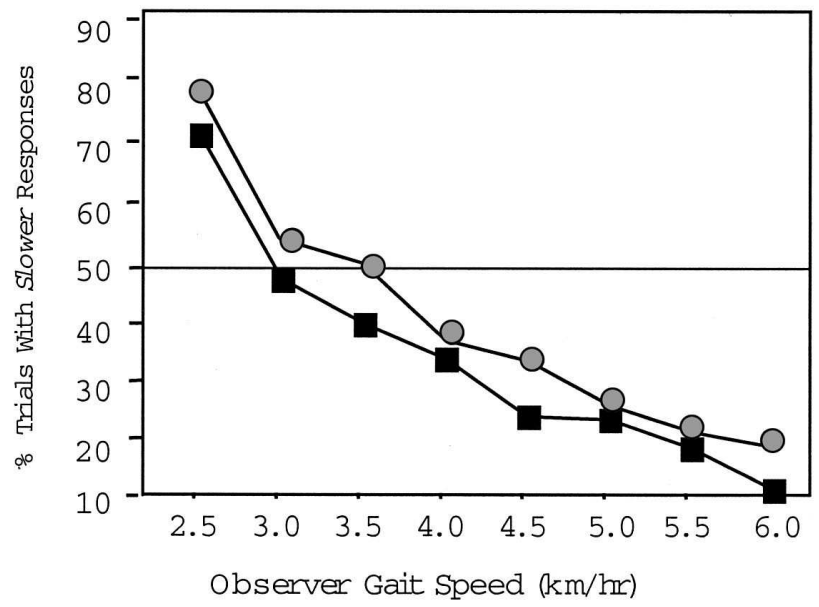

Figure 5. A: Mean discrimination accuracy as a function of treadmill incline and observer gait speed for observers on flat (Experiment 2) and inclined (Experiment 3) treadmills. B: Response bias as a function of treadmill incline and observer gait speed for observers on flat and inclined treadmills in Experiments 2 and 3, respectively. Exp = experiment. 
treadmill. At these speeds, effort (as measured by heart rate) was substantially higher for observers on the inclined treadmill $\left(M_{\text {common }}=140 \mathrm{bpm} ; M_{\text {fast }}=163 \mathrm{bpm}\right)$ than for observers on the flat treadmill $\left(M_{\text {common }}=97 \mathrm{bpm} ; M_{\text {fast }}=117 \mathrm{bpm}\right)$. However, at slow walking speeds $(2.5-3.0 \mathrm{~km} / \mathrm{hr})$, discrimination accuracy did not differ between observers on inclined and flat treadmills, $t(30)=-1.30, p=.21$. At these slow speeds, the difference in effort exerted between observers in the inclined $(M=101 \mathrm{bpm})$ and flat $(M=89 \mathrm{bpm})$ treadmill conditions was smaller. Specifically, at slow speeds, the difference in heart rate was only $12 \mathrm{bpm}$, whereas the difference in heart rate between the inclined and flat conditions was $43 \mathrm{bpm}$ at common walking speeds and $46 \mathrm{bpm}$ at fast walking speeds. Thus, as effort increased when observers walked quickly up an incline, performance accuracy dropped relative to that on a flat treadmill.

We also conducted an analysis of the response biases. The percentage of trials on which observers responded that they walked more slowly than the point-light walker served as the dependent measure. These data are depicted in Figure 5B. This analysis revealed a significant main effect of treadmill incline, $F(1,30)=$ $7.95, p<.01$. Observers walking up the incline were more likely to perceive themselves as walking faster than the point-light walker $(M=66.2 \%)$ than were observers walking on the flat treadmill $(M=59.1 \%)$. This difference was evident at every speed, as indicated by the nonsignificant Observer Gait Speed $\times$ Treadmill Incline interaction, $F(7,210)=0.29, p=.96$. Because observers walked faster than the point-light walker on only half of the trials, these results suggest that increased effort is associated with increased bias in the perception of gait speed.

Although observers on the inclined and flat treadmills adopted identical gait speeds and performed the same gait-speed comparisons, they exhibited distinct perceptual biases. At every speed, observers walking up the incline perceived their own gait speed as faster than the gait speed of the point-light walker. Because walking up an incline requires more effort than walking on a flat surface (Susta et al., 2000), these findings suggest that observer effort substantially influences the perceived relationships between an observer's own gait speed and the gait speed of another person.

\section{Experiment 4: Egocentric Gait-Speed Sensitivity and Observer Fitness}

Motor effort not only varies with external task characteristics, such as ground incline, but also with each individual's intrinsic potential to perform an action. Physically fit individuals exert less effort than unfit individuals in performance of the same motor task (e.g., Sharp, Reed, Sun, Abumrad, \& Hill, 1992). Consistent with this, Bhalla and Proffitt (1999) found physical fitness to be highly correlated with hill-slope estimation $\left(R^{2}=.98\right)$. Specifically, as physical fitness declined, observers provided systematically inflated estimations of hill slope. If physical fitness also influences egocentric gait perception, then unfit observers should exhibit stronger perceptual biases in their egocentric percepts of gait speed than do observers with high levels of physical fitness.

\section{Method}

Twenty-four observers from Rutgers University, Newark, participated in the experiment for either course credit or $\$ 10$. The design and procedure replicated that of the egocentric condition of Experiment 2. The same observer and point-light gait speeds were used. Observers were divided into two groups on the basis of a questionnaire pertaining to their level of physical fitness. The high physical fitness condition contained athletes who exercised five or more times per week. Observers in the low physical fitness condition exercised less than once per week. Thus, the two conditions were identical except for the physical fitness of the observers.

\section{Results and Discussion}

As before, we analyzed percentages of correct gait-speed discrimination with a repeated measures ANOVA. Condition (high vs. low physical fitness) served as a between-subjects variable. Observer gait speed $(2.5-6.0 \mathrm{~km} / \mathrm{hr})$ served as a within-subject variable. The analysis revealed a significant main effect of condition, $F(1,22)=6.11, p<.05$. As can be seen in Figure $6 \mathrm{~A}$, observers in the high physical fitness condition were more accurate $(M=73.7 \%)$ than observers in the low physical fitness condition $(M=64.8 \%)$. This difference was evident at almost every speed,
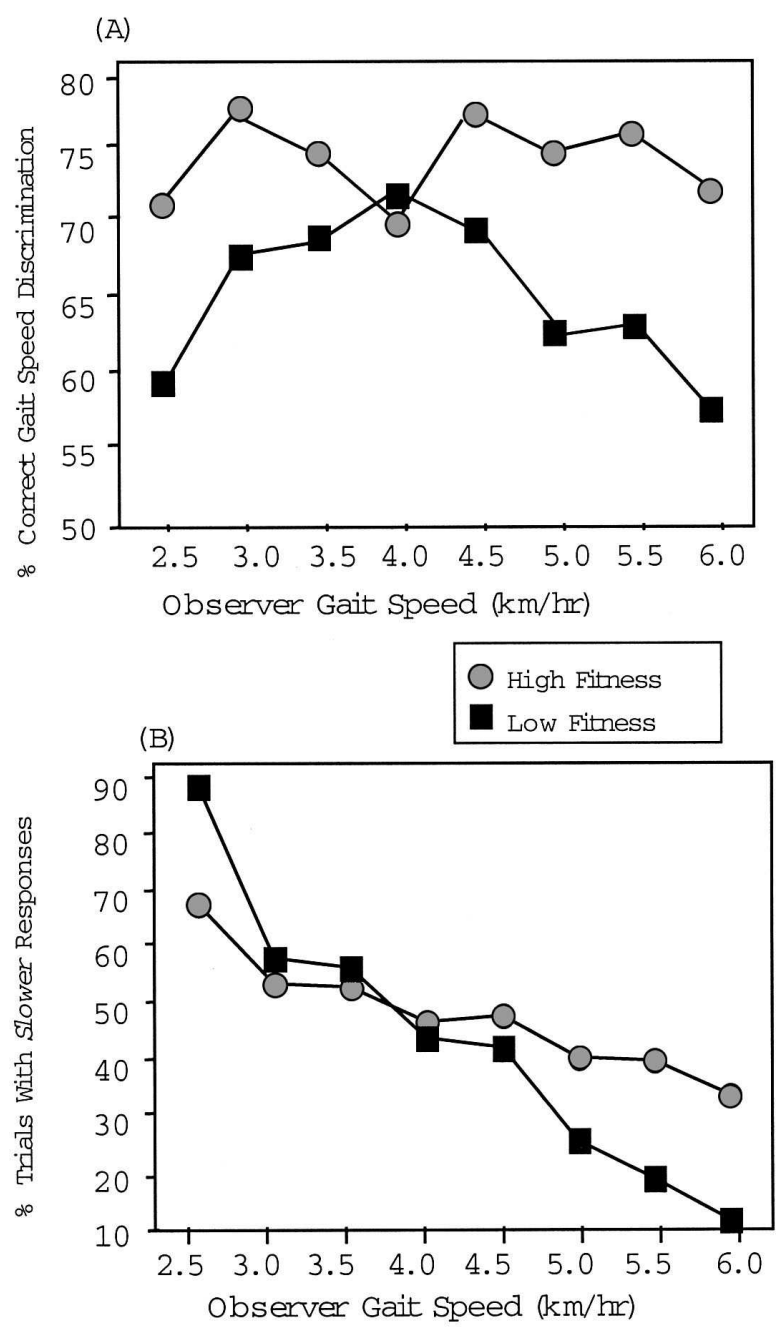

Figure 6. A: Mean discrimination accuracy as a function of fitness level (high vs. low) and observer gait speed for observers in Experiment 4. B: Observer response bias as a function of fitness level and observer gait speed in Experiment 4. 
as indicated by the nonsignificant Observer Gait Speed $\times$ Condition interaction, $F(7,154)=1.57, p=.15$. One-sample $t$ tests revealed that accuracy for observers in both the high physical fitness condition, $t(11)=8.91, p<.01$, and the low physical fitness condition, $t(11)=6.20, p<.01$, was statistically above chance.

As before, we also coded observers' responses for bias as the percentage of trials during which they perceived themselves to be walking slower than the point-light walker. We conducted a repeated measures ANOVA on these bias data, revealing a statistically significant Observer Gait Speed $\times$ Condition interaction, $F(7,154)=5.36, p<.01$. These results can be seen in Figure 6B. The bias to report oneself as walking more slowly than another walker was a function of observer walking speed in both the high physical fitness condition, $F(7,77)=6.70, p<.01$, and the low physical fitness condition, $F(7,77)=27.67, p<.01$. Specifically, as gait speed increased, observers were significantly more likely to report walking faster than the point-light walker. However, although performance in the low physical fitness condition differed significantly from unbiased performance, $t(11)=-2.40, p<.05$, performance in the high physical fitness condition did not, $t(11)=$ $-0.56, p=.59$.

These findings are consistent with research on hill slope perception in which observers of low physical fitness have been found to provide inflated estimations of hill slope relative to observers of high physical fitness (Bhalla \& Proffitt, 1999). In the current experiment, because unfit observers needed to exert more effort to complete the walking task than did highly fit observers, it appears that intrinsic motor effort also influences egocentric perception of other people's movements. Thus, during egocentric action perception, the visual analysis of human movement appears to be significantly altered by input received from an observer's own motor system.

\section{Experiment 5: Exocentric Gait-Speed Sensitivity and Observer Fitness}

In the previous experiment, observers of high and low physical fitness exhibited different performance accuracies and perceptual biases in their egocentric discriminations of gait speed. Although we attributed these differences to motor effort, they could also be attributed to visual differences. Specifically, athletes may perform better simply because they have more visual experience judging relative gait speed. If so, then athletes should also perform exocentric gait-speed comparisons more accurately than do unfit individuals. To test this hypothesis, we had observers of high and low physical fitness perform the exocentric task from Experiment 1 while standing on a stationary treadmill. If visual experience determined the perceptual differences found in the previous experiment, the same differences should be found here.

\section{Method}

Twenty-four undergraduate students from Rutgers University, Newark, participated in this experiment for class credit. The design and procedure of this experiment replicated that of the standing condition in Experiment 1. Specifically, observers stood on a stationary treadmill while judging the gait speeds of two point-light walker displays. There were two groups of observers. Observers in the high physical fitness group exercised at least 5 times per week, whereas observers in the low physical fitness group exercised less than once per week.

\section{Results and Discussion}

The results of this experiment revealed a nonsignificant effect of fitness level on both accuracy, $F(1,22)=1.97, p=.17$, and response bias, $F(1,22)=0.25, p=.62$. Observers of low physical fitness $(M=68.8 \%)$ were just as accurate in judging other-relative gait speed as were observers of high physical fitness $(M=66.8 \%)$. Similarly, observer bias toward perceiving one walker as slower than the other did not depend on the observer's level of physical fitness. One-sample $t$ tests revealed that the performance accuracies of athletes, $t(11)=4.90, p<.01$, and nonathletes, $t(11)=$ $7.96, p<.01$, were both significantly above chance.

The current findings indicate that athletes are no more sensitive to exocentric gait speed than are unfit individuals. This suggests that the performance differences found in Experiment 4 cannot be attributed to differences in visual experience per se. This conclusion is consistent with previous findings that differential visual experience with rare and common gaits does not alter gait-speed sensitivity (Jacobs et al., 2004). Thus, the current results indicate that in Experiment 4, unfit and highly fit individuals performed egocentric gait-speed discrimination tasks differently as a function of differences in the levels of motor effort they exerted.

\section{Experiment 6: Action Coordination and Gait-Speed Perception}

What might account for differences between egocentric and exocentric discriminations of gait speed? One possible explanation concerns the modalities compared. In the previous experiments, egocentric discriminations required visual-motor comparisons, whereas exocentric discriminations of two point-light walkers required visual-visual comparisons. Thus, egocentric and exocentric discriminations of human motion require, by definition, comparisons of different types of information. A second difference concerns the potential for action coordination. Egocentric action perception requires observers to compare, and potentially coordinate, their actions with the actions of other people. Conversely, exocentric perception does not require this.

To test these alternative hypotheses, we had observers perform egocentric comparisons of gait speed under conditions in which action coordination does not normally occur. In the previous experiments, the participants and point-light walkers both faced and walked in the same direction. In the current experiment, participants and point-light walkers faced and walked in opposite directions. Although pedestrians might want to coordinate their gaits with the gaits of people walking along with them, they would not want to coordinate their gaits with the gaits of people walking away from them. Indeed, research has demonstrated that observers are more likely to coordinate their own movements with the movements of another person when the direction of the movements is the same (e.g., Schmidt, Carello, \& Turvey, 1990; Schmidt \& O'Brien, 1997). Similarly, an observer's ability to perform a movement is inhibited by observation of an incongruent motion being performed (Kilner et al., 2003). If gait-speed perception depends on the potential for action coordination, then gait-speed discrimination should vary as a function of whether 
observers and point-light walkers face and walk in the same direction or in opposite directions. That is, the same stimulus might be judged differently depending on an observer's potential ability to interact with it. Conversely, if modality differences account for previously identified differences between egocentric and exocentric perception of gait speed, then direction of facing should have no impact on gait-speed discrimination.

\section{Method}

Sixteen undergraduate students from Rutgers University, Newark, participated in this experiment for course credit. The design and procedure of this experiment replicated that of the egocentric condition in Experiment 2. As before, observers walked on the same treadmill while judging whether they walked faster or slower than a point-light walker. As before, on each trial, the flat treadmill was set at one of the same eight speeds ranging from 2.5 to $6.0 \mathrm{~km} / \mathrm{hr}$. Again, the speed of the point-light walker's gait was 0.5 $\mathrm{km} / \mathrm{hr}$ faster or slower than the observer's own gait speed. The only difference between this experiment and the egocentric condition of Experiment 2 was the direction in which the point-light walker faced. In Experiment 2, the point-light walker and the observer both faced in the same direction. As illustrated in Figure $3 \mathrm{C}$, in the current experiment, the observer and the point-light walker faced in opposite directions. All other aspects of this experiment were identical to those of the egocentric condition of Experiment 2

\section{Results and Discussion}

A one-sample $t$ test revealed that observers in this experiment discriminated gait speed at a level well above chance $(M=$ $73.9 \%), t(15)=8.64, p<.01$. To determine whether performance varied as a function of facing direction, we performed a repeated measures ANOVA with condition (same direction vs. opposite direction) as a between-subjects variable. Observer gait speed served as a within-subject variable. Although overall accuracy was equivalent when observers and point-light walkers faced in the same $(M=71.3 \%)$ and in different $(M=74.4 \%)$ directions, $F(1$, $30)=1.07, p=.31$, there was a significant Observer Gait Speed $\times$ Condition interaction, $F(7,210)=2.85, p<.01$. Specifically, although performance accuracy in the same direction condition peaked at common walking speeds, performance accuracy in the opposite direction condition conformed to Weber's law. These differences are illustrated in Figure 7A. An analysis of the response bias data revealed a significant main effect of condition, $F(1,30)=26.15, p<.01$, and a significant Observer Gait Speed $\times$ Condition interaction, $F(7,21)=21.79, p<.01$. Observers in the opposite direction condition $(M=60.7 \%)$ were more likely to perceive themselves as walking slower than were observers in the same direction condition $(M=40.9 \%)$. Moreover, the effect of observer gait speed on response bias varied with direction of facing. As depicted in Figure 7B, when observers and pointlight walkers faced in the same direction, response biases varied systematically with observer gait speed. Conversely, when observers and point-light walkers faced in opposite directions, response biases were independent of observer gait speed.

These results demonstrate that the potential for action coordination plays a critical role in the visual analysis of human movement. When point-light actors and observers walked in the same direction, and the potential for action coordination was high, sensitivity biases were speed dependent. Conversely, when point-
(A)

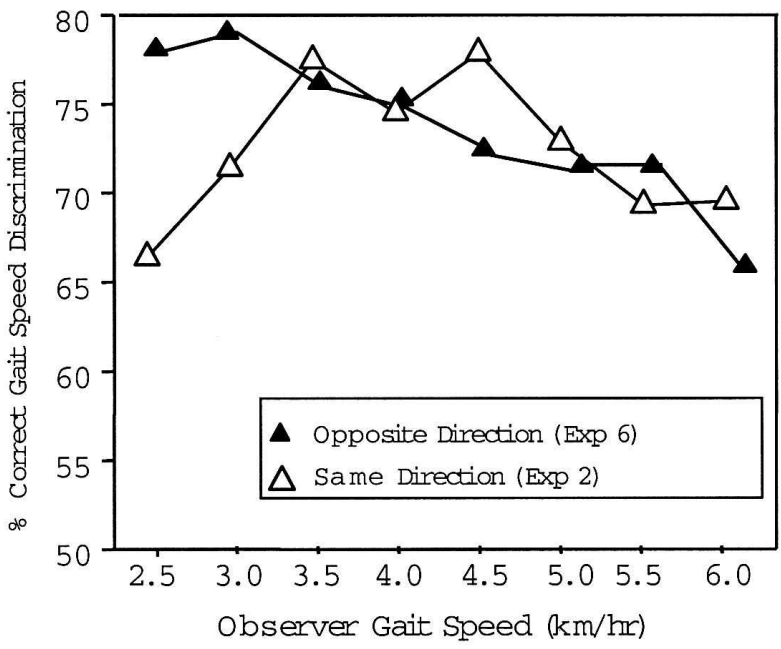

(B)

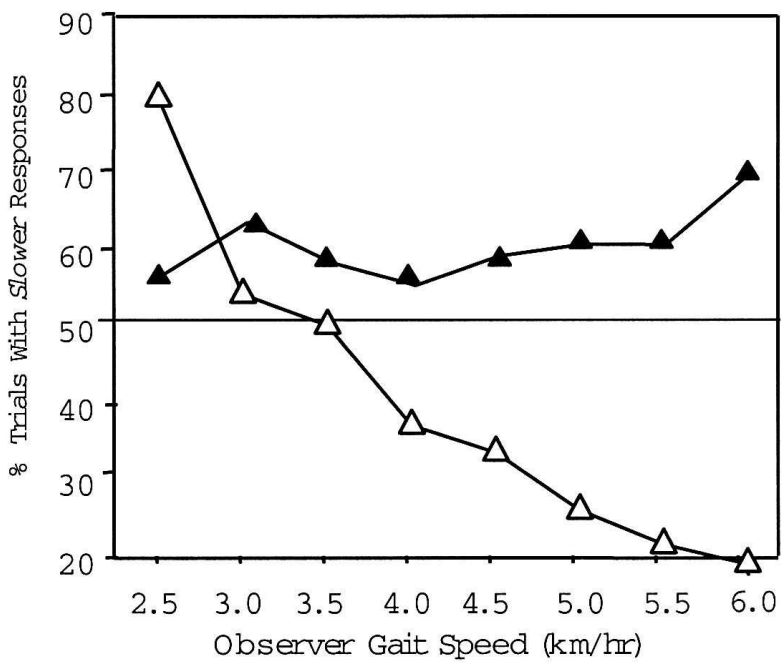

Figure 7. A: Mean egocentric gait-speed discrimination accuracy as a function of direction of facing (opposite vs. same) and observer gait speed in the same direction condition of Experiment 2 and the opposite direction condition of Experiment 6. Performance depended on direction of facing. B: Observer discrimination bias as a function of direction of facing and observer gait speed in Experiments 2 and 6. Discrimination bias plotted as a function of facing direction and gait speed. Exp $=$ experiment.

light actors and observers walked in opposite directions, and the potential for action coordination was low, biases in gait-speed sensitivity were speed independent. Thus, the current findings suggest that the potential for action coordination significantly influences how observers analyze gait speed.

\section{General Discussion}

Decades' worth of research on the visual perception of biological motion has demonstrated that observers have an impressive ability to analyze and interpret the actions of other people. Traditionally, this research has focused on how stationary observers analyze the actions of others. Unlike the observers in these labo- 
ratory experiments, observers in the real world frequently analyze the movements of other people for the purpose of action coordination. This process requires moving observers to compare their own actions with the actions of others. We examined the effects of action production on biological motion perception in six psychophysical experiments.

To determine whether observer movement affects action perception, we had observers in Experiment 1 walk, stand, or cycle as they compared the relative gait speeds of two point-light walkers. Walking interfered with walking perception, suggesting that action production interferes with the perception of similar actions. In Experiment 2, we compared egocentric and exocentric gait-speed perception to determine whether walking observers use similar visual-motor processes for those two types of analyses. Gait-speed perception was found to depend on observers' motor experience in the egocentric, but not the exocentric, condition. In Experiments 3 and 4 , we further investigated the nature of this motor dependency through variations in treadmill incline and physical fitness. In both experiments, egocentric gait-speed discrimination depended on the observer's motor effort. Experiment 5 was designed to determine whether perceptual differences between observers of high and low physical fitness resulted from their visual or their motor experience. The finding that athletes and nonathletes differ in their performance of egocentric, but not exocentric, gait-speed discriminations suggests that motor effort is key. Finally, in Experiment 6, observers compared their own gait speed to the gait speed of a point-light actor walking in the opposite direction. The results of this experiment suggest that the potential for action coordination may be responsible for the differences found between self-relative and other-relative gait-speed perception.

Clearly, our experiments did not mimic all aspects of real-world action coordination. For example, locomotor differences exist between treadmill and ground walking. One such difference is a slight increase in step frequency with treadmill walking (e.g., Stolze et al., 1997). Although such differences may limit the absolute ecological validity of our experiments, our approach nonetheless represents an advance over previous studies in that we asked participants to perform a real-world behavior-namely, to analyze human motion for the purpose of action coordination. Furthermore, the requirement that participants perform these comparisons while walking on a treadmill was useful because the people depicted in the point-light displays also walked on a treadmill. Thus, any motor adjustments that occurred from walking on a treadmill occurred for both the observers and the point-light walkers.

Another limitation of the current work is our restriction to speed rather than velocity (speed + direction) judgments. Previous studies have examined how people navigate around stationary and moving objects (e.g., Cutting et al., 1995; Fajen \& Warren, 2003). Because the current experiments involved treadmill walking, we focused exclusively on the perception of constant-direction walking. As such, we specifically studied perception under collisionfree conditions. Because the goal of our work was to extend biological motion research to moving observers, rather than to extend optic flow research to peopled environments, we adopted the most frequently used methodologies in biological motion research - namely, the presentation of fixed sagittal views of pointlight walkers. In future studies, we hope to vary both the speed and the direction of life-sized walkers in large environments so that the influence of collision constraints on walking perception can be systematically determined.

The current set of experiments provides important advances to the understanding of human movement perception. First, action production can interfere with action perception. Indeed, in Experiment 1, observers' own walking movements interfered with their sensitivity to the gaits of other people. These findings support previous conclusions that observers use motor representations during their visual analyses of other people's actions (Prinz, 1997; Reed \& Farah, 1995; Shiffrar \& Pinto, 2002; Viviani \& Stucchi, 1992). The finding from Experiment 1 that cycling does not influence walking perception suggests that interactions between action production and action perception occur maximally for similar movements. This conclusion is consistent with previous work on action identification (Knoblich \& Flach, 2001, 2003; Loula et al., 2005). The current results also indicate that theories of biological motion perception based on data from stationary observers will be difficult to extrapolate to biological motion perception under real-world conditions in which observers effortfully and purposefully move.

Second, observers use motor information in qualitatively different ways when they perform self-relative and other-relative action comparisons. Experiments 1-5 demonstrated that egocentric action perception is influenced by both prior and current motor experiences, whereas exocentric action perception is not. Specifically, observers' ability to perform egocentric gait-speed comparisons was best when they walked at frequently adopted gait speeds. Furthermore, when observers exerted increased or decreased motor effort, their judgments of self-relative gait speed became systematically biased. This occurred when effort was manipulated by an observer's own level of physical fitness and when it was manipulated by variations in the speed and incline of the treadmill on which he or she walked. Such a dependence of motor effort on visual perception is consistent with previous studies of slant and distance perception (Bhalla \& Proffitt, 1999; Proffitt et al., 1995, 2003). In sum, the current results suggest that each observer's current motor effort influences his or her self-relative perception of biological motion. Thus, self-relative action perception appears to depend on previous and current motor experience in ways that other-relative action perception does not.

An alternative account of our findings is that visual experience, rather than motor experience, constrains egocentric action perception. For instance, athletes may have been more accurate in their egocentric judgments of gait speed simply because they had more experience carefully watching the actions of others. Experiment 5 addressed this possibility through an examination of speed sensitivity in other-relative judgments by athletes and unfit observers. Because athletes did not show increased sensitivity to gait speed under other-relative conditions, we conclude that motor effort defines our current effects. This is not to say that biological motion perception is independent of visual experience. Indeed, studies of identity perception suggest that visual experience can influence how well observers identify other people from their actions (Jacobs et al., 2004; Loula et al., 2005).

Finally, the results of Experiment 6 indicate that performance differences between the egocentric and exocentric gait-speed perception depend on the potential for action coordination. When observers made self-relative speed discriminations of people walking away from them, their performance approximated that found 
for other-relative speed judgments. Motor research has shown that an individual's ability to coordinate his or her actions with the actions of another person decreases as the directional congruency of the motions decreases (Schmidt et al., 1990; Schmidt \& O'Brien, 1997). Thus, performance in our perceptual task was associated with the potential to coordinate one's own actions accurately with the actions of other people. Such an association suggests that the potential for action coordination influences the types of perceptual and motor processes that are invoked during human movement perception.

In sum, the current set of experiments suggests that moving and stationary observers can perceive human movement very differently. Furthermore, when observers move, their egocentric and exocentric comparisons of human actions can differ. These differences depend on the potential for action coordination. When selfrelative action coordination was possible, visual analyses of gait speed depended on an observer's own gait speed, exertion level, and prior walking experience. Conversely, when the same gaitspeed discriminations were performed under conditions in which action coordination was not possible or required, gait-speed perception became independent of an observer's gait speed, effort, and prior walking experience. These findings suggest that normally moving observers perform visual analyses of human movement that are distinct from the visual analyses performed by stationary, noninteractive observers. Thus, the development of comprehensive theories of biological motion perception must take into account motor and ecological constraints as well as visual processes.

\section{References}

Adolph, K. E. (2000). Specificity of learning: Why infants fall over a veritable cliff. Psychological Science, 11, 290-295.

Bertenthal, B. I., \& Pinto, J. (1994). Global processing of biological motions. Psychological Science, 5, 221-225.

Bhalla, M., \& Proffitt, D. (1999). Visual-motor recalibration in geographical slant perception. Journal of Experimental Psychology: Human Perception and Performance, 25, 1076-1096.

Brownlow, S., Dixon, A. R., Egbert, C. A., \& Radcliffe, R. D. (1997). Perception of movement and dancer characteristics from point-light displays of dance. Psychological Record, 47, 411-421.

Cutting, J. E., Moore, C., \& Morrison, R. (1988). Masking the motions of human gait. Perception \& Psychophysics, 44, 339-347.

Cutting, J. E., Vishton, P. M., \& Braren, P. A. (1995). How we avoid collisions with stationary and moving obstacles. Psychological Review, 102, 627-651.

Diedrich, F. J., \& Warren, W. H. (1998). The dynamics of gait transitions: Effects of grade and load. Journal of Motor Behavior, 30, 323-343.

Dittrich, W. H. (1993). Action categories and the perception of biological motion. Perception, 22, 15-22.

Dittrich, W. H., Troscianko, T., Lea, S. E. G., \& Morgan, D. (1996). Perception of emotion from dynamic point-light displays represented in dance. Perception, 25, 727-738.

Fajen, B. R., \& Warren, W. H. (2003). Behavioral dynamics of steering, obstacle avoidance, and route selection. Journal of Experimental Psychology: Human Perception and Performance, 29, 343-362.

Gibson, E. J., \& Pick, A. D. (2000). An ecological approach to perceptual learning and development. New York: Oxford University Press.

Gibson, J. J. (1986). The ecological approach to visual perception. Hillsdale, NJ: Erlbaum.

Haggard, P., Cockburn, J., Cock, J., Fordham, C., \& Wade, D. (2000). Interference between gait and cognitive tasks in a rehabilitating neuro- logical population. Journal of Neurology, Neurosurgery, and Psychiatry, 69, 479-486.

Hreljac, A. (1993). Preferred and energetically optimal gait transition speeds in human locomotion. Sport Exercise, 25, 1158-1162.

Inman, V. T., Ralston, H. J., \& Todd, F. (1981). Human walking. Baltimore: Williams \& Wilkins.

Jacobs, A., Pinto, J., \& Shiffrar, M. (2004). Experience, context, and the visual perception of human movement. Journal of Experimental Psychology: Human Perception and Performance, 30, 822-835.

Johansson, G. (1973). Visual perception of biological motion and a model for its analysis. Perception \& Psychophysics, 14, 201-211.

Johansson, G. (1976). Spatio-temporal differentiation and integration in visual motion perception: An experimental and theoretical analysis of calculus-like functions in visual data processing. Psychological Research, 38, 379-393.

Kilner, J. M., Paulignan, Y., \& Blakemore, S. J. (2003). An interference effect of observed biological movement on action. Current Biology, 13, $522-525$.

Knoblich, G., \& Flach, R. (2001). Predicting effects of actions: Interactions of perception and action. Psychological Science, 12, 467-471.

Knoblich, G., \& Flach, R. (2003). Action identity: Evidence from selfrecognition, prediction, and coordination. Consciousness \& Cognition, 12, 620-632.

Loomis, J. M., Da Silva, J. A., Philbeck, J. W., \& Fukusima, S. S. (1996). Visual perception of location and distance. Current Directions in Psychological Science, 5, 72-77.

Loula, F., Prasad, S., Harber, K., \& Shiffrar, M. (2005). Recognizing people from their movement. Journal of Experimental Psychology: Human Perception and Performance, 31, 210-220.

Prinz, W. (1997). Perception and action planning. European Journal of Cognitive Psychology, 9, 129-154.

Proffitt, D. R., Bhalla, M., Gossweiler, R., \& Midgett, J. (1995). Perceiving geographical slant. Psychonomic Bulletin \& Review, 2, 409-428.

Proffitt, D. R., Stefanucci, J., Banton, T., \& Epstein, W. (2003). The role of effort in perceiving distance. Psychological Science, 14, 106-112.

Reed, C. L., \& Farah, M. J. (1995). The psychological reality of the body schema: A test with normal participants. Journal of Experimental Psychology: Human Perception and Performance, 21, 334-343.

Rizzolatti, G., Fadiga, L., Gallese, V., \& Fogassi, L. (1996). Premotor cortex and the recognition of motor actions. Cognitive Brain Research, 3, 131-141.

Ruby, P., \& Decety, J. (2001). Effect of subjective perspective taking during simulation of action: A PET investigation of agency. Nature Neuroscience, 4, 546-550.

Runeson, S., \& Frykholm, G. (1981). Visual perception of lifted weight. Journal of Experimental Psychology: Human Perception and Performance, 7, 733-740.

Runeson, S., \& Frykholm, G. (1983). Kinematic specification of dynamics as an informational bias for person-and-action perception: Expectation, gender recognition, and deceptive intent. Journal of Experimental Psychology: General, 112, 585-615.

Schmidt, R. C., Carello, C., \& Turvey, M. T. (1990). Phase transitions and critical fluctuations in the visual coordination of rhythmic movements between people. Journal of Experimental Psychology: Human Perception and Performance, 16, 227-247.

Schmidt, R. C., \& O’Brien, B. (1997). Evaluating the dynamics of unintended interpersonal coordination. Ecological Psychology, 9, 189-206.

Sharp, T. A., Reed, G. W., Sun, M., Abumrad, N. N., \& Hill, J. O. (1992). Relationship between aerobic fitness level and daily energy expenditure in weight-stable humans. American Journal of Physiology, 263, 121128.

Shiffrar, M., \& Pinto, J. (2002). The visual analysis of bodily motion. In W. Prinz \& B. Hommel (Eds.), Common mechanisms in perception and 
action: Attention and performance XIX (pp. 381-399). New York: Oxford University Press.

Stevens, J. A., Fonlupt, P., Shiffrar, M., \& Decety, J. (2000). New aspects of motion perception: Selective neural encoding of apparent human movements. NeuroReport, 11, 109-115.

Stolze, H., Kuhtz-Buschbeck, J. P., Mondwurf, C., Boczek-Funcke, A., Joehnk, K., Deuschl, G., \& Illert, M. (1997). Gait analysis during treadmill and overground locomotion in children and adults. Electroencephalography and Clinical Neurophysiology: Electromyography and Motor Control, 195, 490-497.

Susta, D., Minetti, A., Moia, C., \& Ferretti, G. (2000). Energy costs of walking and running on extreme uphill and downhill slopes. Journal of Physiology, 523, 227.

Thornton, I., Pinto, J., \& Shiffrar, M. (1998). The visual perception of human locomotion across space and time. Cognitive Neuropsychology, $15,535-552$.

Thornton, I. M., Rensink, R. A., \& Shiffrar, M. (2002). Active versus passive processing of biological motion. Perception, 31, 837-853.

Viviani, P., \& Stucchi, N. (1992). Biological movements look constant: Evidence of motor-perceptual interactions. Journal of Experimental Psychology: Human Perception and Performance, 18, 603-623.

Vogeley, K., \& Fink, G. R. (2003). Neural correlates of the first-personperspective. Trends in Cognitive Science, 7, 38-42.

Received November 21, 2003 Revision received May 24, 2004 Accepted August 9, 2004

\section{American Psychological Association SUbSCRIPTION Claims Information}

Today's Date:

We provide this form to assist members, institutions, and nonmember individuals with any subscription problems. With the appropriate information we can begin a resolution. If you use the services of an agent, please do NOT duplicate claims through them and directly to us. PLEASE PRINT CLEARLY AND IN INK IF POSSIBLE.

PRINT FULL NAME OR KEY NAME OF INSTITUTION

\begin{tabular}{llll}
\hline ADDRESS & & \\
\hline CITY & STATE/COUNTRY & ZIP
\end{tabular}

YOUR NAME AND PHONE NUMBER

TITLE
MEMBER OR CUSTOMER NUMBER (MAY BEFOUND ON ANY PAST ISSUE LABEL)

DATE YOUR ORDER WAS MAILED (OR PHONED)

PREPAID CHECK CHARGE

CHECK/CARD CLEARED DATE:

(If possible, send a copy, front and back, of your cancelled check to help us in our research of your claimn.)

ISSUES: DAMAGED

VOLUME OR YEAR

Thank you. Once a claim is received and resolved, delivery of replacement issues routinely takes 4-6 weeks.

(TO BE FILLED OUT BY APA STAFF)

DATE RECEIVED:

ACTION TAKEN:

STAFF NAME:
DATE OF ACTION:

INV. NO. \& DATE:

LABEL NO. \& DATE:

Send this form to APA Subscription Claims, 750 First Street, NE, Washington, DC 20002-4242

PLEASE DO NOT REMOVE. A PHOTOCOPY MAY BE USED. 